CHAPTER 10

\title{
Use and Abuse of Citations
}

\author{
Robert West, Kerstin Stenius and Tom Kettunen
}

\section{Introduction}

Research output in the form of articles, books, and book chapters exists to be used by other researchers to inform subsequent research, influence policy decisions, and improve clinical practice. Authors need to consider how to make appropriate use of their previous publications and the work of others and how to ensure that their own work will be used appropriately.

A research article, book, policy document, or treatment manual should refer to other writing that is relevant to its message. Citation is the formal vehicle for doing this. It involves explicit reference to a piece of research output that, in principle, can be anything from an article in a journal to a website. Conventions applying to citation practice regulate the transmission of information, and citation conventions vary from one research field to another. The following text focuses primarily on what might be termed cumulative research in which the goal is to accumulate enduring knowledge and understanding.

There are two main types of citation (Box 10.1). In this chapter we use the term referential citation to refer to the situation in which a piece of research output (which may be empirical or conceptual) is being used for what it contributes to the field. The term critical citation is used when the citing piece points to what is considered a flaw in some research output.

The citation serves one or more essential functions: It enables the reader to examine the cited work to check the veracity of a statement that it is being used to support or the correctness of the use of a concept or interpretation of a process. When citing in support of a statement being made in one's own article, it also acknowledges the contribution made by the cited work. Both

\section{How to cite this book chapter:}

West, R, Stenius, K and Kettunen, T. 2017. Use and Abuse of Citations. In: Babor, T F,

Stenius, K, Pates, R, Miovský, M, O’Reilly, J and Candon, P. (eds.) Publishing Addiction Science: A Guide for the Perplexed, Pp. 191-205. London: Ubiquity Press. DOI: https://doi.org/10.5334/bbd.j. License: CC-BY 4.0. 
the verification function and the acknowledgement function are important. One may also use citations to document how a political debate, historical process, or specific concept has developed and has been defined. We can call this the documentation function. ${ }^{1}$

Regarding the verification function and the documentation function, the scope for intentional and unintentional distortion of research through unfounded assertions or misleading statements is enormous. In principle, every nonobvious factual claim should be supported in some way, either by citing direct evidence or by tracing a link through citations and/or inference to that evidence. Similarly every hypothesis, conceptual analysis, or statement of a theoretical position that is not advanced for the first time in a given article should trace a link to its source. Citations offer the readers an opportunity to determine for themselves whether the original source of a claim was justified and whether that claim is being accurately represented in the current piece.

Regarding the acknowledgement function, it is right and proper that researchers should receive credit for their work, and citation is the primary

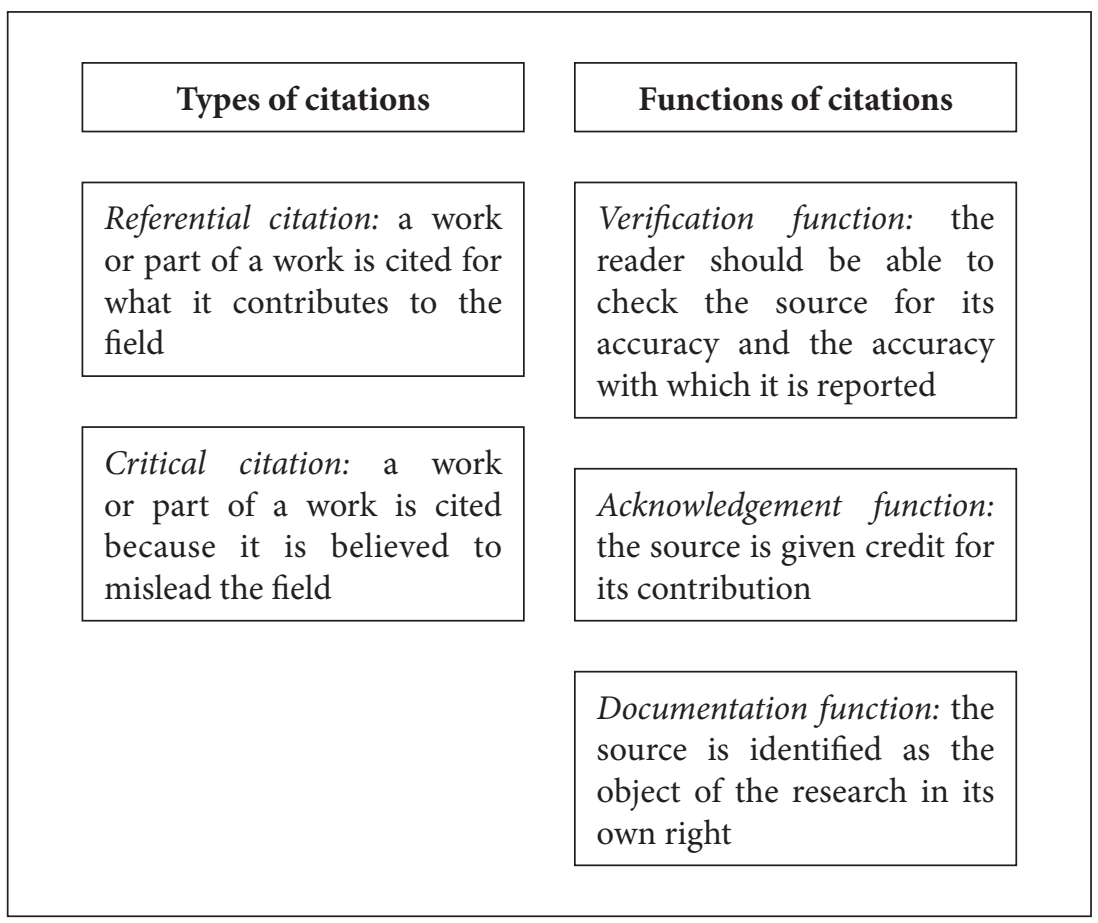

Box 10.1: Types and functions of citations. 
means by which this is achieved. This is not merely a matter of etiquette: Employment and promotion of individual researchers are built on reputation, and citations play a crucial role in this. The institutions that employ researchers achieve kudos and in many cases funding on the basis of the reputations of their employees. Moreover, charities and government bodies that fund research must receive credit for the work they support. Their own income may well depend on it.

\section{Deviations from Ideal Citation Practice}

Citation practice often falls far short of the ideal (for a discussion, see Reyes, 2001). There are a number of sources one may use to find out about good practice in the use of citations in systematic reviews (e.g., Bannigan et al., 1997; Chalmers et al., 1993; Cook et al., 1995; Moher et al., 2009; Petticrew et al., 2008; Reeves et al., 2002; Stroup et al., 2000; Sutton et al., 1999; see also Chapter 9). Use of citations in less formal reviews, such as to introduce research reports, is subject to greater variability. The following paragraphs examine common deviations from ideal practice (see also Table 10.1).

\section{Selective Citation through need for Conciseness}

A legitimate reason to depart from ideal practice arises from the need for conciseness. Many times in a given field, a large number of studies may be cited in support of a given statement. In the absence of other constraints, the acknowledgement function might dictate that all relevant studies are cited. However, this would be impracticable. This raises the question of which article or articles to cite. There is a case for citing what we might call the discovery article: the first article to record the finding. However, this may be impossible to determine. Moreover, it may not represent the most robust support for the assertion in question. There is a case for citing a review article (an article that summarizes the research on a specific topic). This has the advantage of pointing the reader, at least indirectly, to a body of work rather than one or two studies that might be unrepresentative. The disadvantages are (a) the increased danger of misrepresentation because of hearsay and (b) failure to acknowledge the contribution of the original source.

A possible rule of thumb in determining policy relating to a specific finding is to aim to cite the discovery piece and no more than five other original sources that testify to the generality of the finding, unless there is an authoritative and noncontentious review that can be cited instead. When referring to a conceptual or theoretical exposition, the first major presentation of the current version should be used. 


\section{Selective Citation in Support of a Viewpoint}

A common bias in reporting the literature is to select only (or primarily) studies that support a given hypothesis or idea (viewpoint citation). This is harder to avoid and to detect than one might imagine. If there were a well-defined body of literature that examined a particular hypothesis, and numerous high-quality studies conflicting with the hypothesis were ignored in a review, that would amount in the eyes of some to scientific misconduct. A reader who was not familiar with the area would be misled as much as if the author had fabricated data.

Less straightforward is the case where there are doubts about the methodological adequacy of conflicting studies. For example, studies that fail to detect the effect of an intervention may be small or involve inadequate implementation of the intervention. Unless one is explicitly attempting a comprehensive review in which there is the space to explore these issues, the citing author has to make a judgement about how far to go in ignoring weak studies. Given the realistic possibility that the citing author is not wholly disinterested in the matter, it is good practice to alert the reader to conflicting findings and make a brief comment about the weight that might be attached to these and why.

Even less straightforward is the case in which it is extremely difficult to determine what the corpus of findings on the topic is. This can happen for findings that typically do not form the main focus of articles. In the smoking literature, for example, it has been noted and is widely believed that depressed smokers are less likely to succeed in attempts to stop than are non-depressed smokers. There are certainly studies showing such an association (Covey, 1999; Glassman et al. 1990). However, often buried in reports of clinical trials and other studies are numerous reports of failures to find such an association, and indeed one metaanalysis has reported no association (Hitsman et al. 2003). There is no doubt that there are even more instances in which the association has been looked for and not found, with no subsequent report being made. At the very least, scientific prudence dictates that findings that are susceptible to this kind of phenomenon be cited with suitable caveats.

\section{Selective Citation to Enhance Reputation}

Self-citation or the citation of colleagues with a view to enhancing one's own or the colleague's reputation (reputation citation) is clearly unacceptable. It distorts science and the process of science and is personally damaging to individuals in less-powerful positions or to those who do not engage in that practice (see e.g. Fowler et al., 2007). One may debate how widespread this practice is, but there can be little doubt that self-serving bias runs at some level throughout the scientific literature (see e.g. Aksnes, 2003). 
Self-citation can also apply to journals (articles in journals tending to cite articles from the same journal). This may arise for reasons other than reputation citation, some of which may be legitimate, but it can distort the literature. One study found significant differences in self-citation rates among journals of anesthesiology (Fassoulaki et al., 2000).

It may be thought that a bias of this kind would be easily detected and an appropriate correction could be applied. However, this is probably optimistic. It is not unreasonable that one's own name should feature prominently in a reference list given that one's research is presumably to some degree programmatic. A similar principle would hold true for one's close colleagues. It can be difficult therefore to tell when this bias is operating.

\section{Selective Citation for Convenience}

Using citations that are easy to find or that happen to have come to the attention of the author is not good practice but is probably very common. There may be many ways in which convenience citation can distort the literature. Insofar as more accessible articles may not represent the literature, use of convenience citations would create a biased impression. Searchable electronic databases, in principle, could mitigate the problem, but they can also lead to their own kind of distortion. It would be expected that they would favor English-language articles in journals indexed by the main databases. One would also expect more recent articles to gain preference because of the way that electronic databases sort the results of searches. Convenience citation would also be expected to favor the more popular journals. One might argue that this is no bad thing because it would be the better articles that would in general find their way into these journals. However, this is not necessarily so.

\section{Selective Citation by Country of Origin}

It goes without saying that a tendency to cite articles simply because they are from one's own country of origin is not good practice. Many researchers are under the impression that this occurs, however. Naturally, the greatest suspicion falls on the U.S. as the main producer of research output, and many non-U.S. researchers can probably recount cases where a U.S. author has cited predominantly or exclusively U.S. references, even when more appropriate ones from other countries exist. In fact, this bias has been found among both U.K. and U.S. researchers publishing in major medical journals (Campbell, 1990; Grange, 1999). Another study found that North American journals cite North American journals to a greater extent than did journals from other regions (Fassoulaki et al., 2000), but the opposite has also been found (Lancho Barrantes et al., 2012; Pasterkamp et al., 2007). 


\section{Citing Inaccessible Sources}

It is quite common for authors to cite conference papers or their abstracts, submitted articles, in-house papers, or unpublished reports (the so-called gray literature). The problem with this kind of citation is that it does not fulfill the verification function of citation. Therefore, it is generally to be discouraged. There may be cases where it is the only option and important in fulfilling the acknowledgement or documentation role, but if this is not obvious, the use should be justified. If that citation is more than a few years old, the use becomes increasingly problematic. It is often reasonable to presume that if it is article or abstract and the finding was robust, it would have found its way into the peer-reviewed literature.

It is becoming common to cite websites. This is reasonable but will pose increasing problems over time as websites move or become inaccessible. In general, for any statement intended to have lasting significance, this practice is best avoided until a system is devised for ensuring the longevity of web-based scientific literature. In policy analyses or descriptions of historical processes, though, references to sources such as websites and government documents may be a key part of the research process.

\section{Citing Unevaluated Sources}

When a citation is used to support a substantive statement, the implication is that the cited reference reports evidence in support of that statement. Inadequate though it is, peer review is the primary gatekeeper for this kind of report.

\begin{tabular}{|l|l|}
\hline Convenience citation & selects citation material that is easy to find \\
\hline Discovery article & the article that first puts forward a new concept \\
\hline Gray literature & $\begin{array}{l}\text { unpublished matter, such as conference presenta- } \\
\text { tions, submitted articles, and in-house papers and } \\
\text { reports }\end{array}$ \\
\hline Publication lag & $\begin{array}{l}\text { the time between an article's acceptance by a } \\
\text { journal and its publication }\end{array}$ \\
\hline Reputation citation & $\begin{array}{l}\text { cites a work or part of a work with a view to } \\
\text { enhancing one's own reputation or that of a } \\
\text { colleague }\end{array}$ \\
\hline Review article & $\begin{array}{l}\text { an article that summarizes the research on a } \\
\text { specific topic }\end{array}$ \\
\hline Viewpoint citation & $\begin{array}{l}\text { cites a work or part of a work because it supports } \\
\text { a given hypothesis or idea }\end{array}$ \\
\hline
\end{tabular}

Table 10.1: Terminology related to deviations from ideal citation practice. 
However, it is commonplace for statements of fact to be supported by citations to book chapters, letters, conference presentations, abstracts, opinion pieces, and other material that has not been peer reviewed. Although in principle readers can track down the source and make their own evaluations, this is often impracticable. The only thing that comes close to a safeguard is that cited work has been through a peer-review process. Within the social sciences, though, even non-peer-reviewed books still remain a main source for new analytical concepts. In some cases, however, the review process for books is as rigorous as the peer-review process for journal articles.

\section{Citing Without Reading}

There is a great temptation to cite a work or part of a work on the strength of a report of what it says without going to the original source. Thus, if an article or a book chapter that we have access to makes a statement that is relevant to our work and cites another article in support of it, it is tempting to repeat the assertion and the citation without reading the original source material. This is clearly unacceptable because of the risk of misrepresentation. Equally, having identified an abstract of an article using an electronic database, an author may be tempted to cite the article without going to the full text. This is risky practice because one has not taken the opportunity to evaluate the research being cited by reading the methods and analyses used.

As a general principle, authors should not make reference to research output without having read and evaluated that output directly.

\section{Overuse of Citations}

Much of the earlier discussion concerned selective use of citations. Quite a common problem is the reverse: providing a long list of citations to support a single statement when fewer would be sufficient. If it is important that the work of the authors of all the various works be acknowledged or if the intention is to provide a comprehensive review, then a long list of citations is appropriate. Otherwise it can make an article unwieldy, and the rule of thumb of selective citation described earlier could be adopted.

\section{Coercive Citation}

During the peer-review process, editors can be tempted to help increase the standing of their journal by encouraging authors to add more citations to the journal, without specifying relevant articles or indicating where more references are needed. This practice is sometimes referred to as coercive self-citation. 
Coercive citation is inappropriate as it undermines the integrity of academic publishing and it should be resisted by both authors and editors. Unfortunately, the practice is widespread and strategic. One study found that around $20 \%$ of academics in business disciplines, economics, sociology and psychology have experienced coercive citation practices (Wilhite \& Fong, 2012). The study also found that editors soliciting superfluous citations are more likely to target manuscripts written by few authors, preferably by scholars of lower academic rank.

\section{Getting Cited}

All the above should suggest that the process of citation is subject to considerable bias, and, although there is a duty on researchers to minimize this, it is unlikely that bias will ever be eliminated. This being said, if one is writing an article that one believes is important, it would seem reasonable to try to ensure that it is drawn to the attention of its intended audience, and that means being cited. The choice of journal is obviously of relevance (see Chapter 3). And it may not be the most prestigious journal that offers the best chance but, rather, the best-quality specialist journal. The most prestigious journals tend to be generalist and, as such, may not be routinely read by many potential users of the research. Whatever outlet one uses for one's research, it can often be a good idea to take other steps to publicize the findings. Some researchers email or send copies of their articles to colleagues. One might post reference to them on listserves or publicize them on social media. With increasing use of Open Access, full text can often be made available on demand. Conference presentations and websites are also potentially useful sources of publicity.

\section{Citation Indexes}

We mentioned earlier that citations are often used as a marker of quality. There is a presumption that the more often an article is cited, in some sense the better it is. This extends to journals, for which the single most widely used measure of quality is the impact factor. The impact factor for a journal in a given year is calculated as the average number of citations in that year to articles in the preceding two years. Thus, if a journal published 50 articles in 2013 and 2014 and there were 100 citations to these articles in 2015, the journal's impact factor for 2015 would be 2.0. Citations of authors to their own work are included. Therefore, clearly the more prolific an author is and the more that authors cite their own work, the more useful those authors are to a journal wanting to maximize its impact factor.

Researchers are often judged by the citation counts of their articles and by the impact factors of the journals in which they publish. Funding decisions in many institutions are based in part on members of those institutions publishing 
in "high-impact" journals. Unfortunately there are many problems associated with using citation counts as a marker of quality and even more with using the impact factor (Hecht et al., 1998; Jones, 1999; Opthof, 1997; Seglen, 1997; Semenzato \& Agostini, 2000). Some researchers have suggested that it may be possible to use citation counts and impact factor with appropriate caveats and corrections (Braun, 2003; Fassoulaki et al., 2002; Rostami-Hodjegan \& Tucker, 2001), whereas others have argued that such use should be abandoned (Bloch \& Walter, 2001; Ojasooet al., 2002; Walter et al., 2003).

Regarding citation counts, the various biases in the use of citations discussed earlier should give an indication of the problem with using them as a marker of quality. In addition, it should be recalled that critical citation is quite commonplace. Therefore, an article might be cited precisely because it is weak or misleading. One article examined the association between peer ratings of quality and the numbers of citations between 1997 and 2000 to articles appearing in the journal Addiction in 1997 (West \& McIlwaine, 2002). Although two independent reviewers agreed moderately in their ratings of the articles, the correlation between these ratings and the number of citations was almost zero. One factor that was correlated with citation count was the region of origin of the first author of the article: Articles from English speaking countries received more citations than those from continental Europe, which received more than those from the rest of the world. A larger analysis of citations to articles in emergency medicine revealed that the citation count of articles was predicted to some extent by the impact factor of the journal in which they appeared and to a more limited extent by quality of the articles (Callahamet al., 2002). A further study of citations to articles reporting randomized trials in hepatobiliary disease found a significant association with a positive outcome but no association with adjudged quality (Kjaergard \& Gluud, 2002).

Apart from the biases already discussed, the fact that only a small proportion of predominantly U.S. journals are indexed in Web of Science would lead to a bias, particularly against non-English-speaking countries. One study reported that exclusion of core journals in emergency medicine had led citation counts in the field to remain low despite considerable expansion of the field (Gallagher \& Barnaby, 1998). Another noted that the way to improve the impact factors of journals in dermatology was to increase the number of them indexed by Web of Science (Jemec, 2001). Another bias arises from researchers in some fields, such as biosciences, simply using more citations than researchers in other fields. This will disadvantage authors in low-citing fields, typically the social sciences. Another bias pertains to texts such as editorials, letters and book reviews not being included in the denominator of citable documents. When they are cited, this can distort the impact factors of small-volume journals. For example, journals publishing mostly "noncitable" book reviews can have surprisingly high impact factors (Jasco, 2009). There are a range of other factors that make citation counts potentially misleading as a marker of quality (Box 10.2). 
- Articles are sometimes cited as criticism.

- Articles describing important original studies are often neglected in favor of reviews.

- There is a bias toward citing articles from one's own country or research group or articles that are easily accessible.

- Some fields of study generate more citations than others irrespective of how important the articles are, for example, fields with high levels of activity and mature fields.

- The importance and quality of a work or part of a work may relate to its policy or clinical implications rather than its use by other researchers.

- Other researchers may fail to grasp the importance of a work or part of a work.

- The citation indexes are biased toward U.S. and other English-language journals.

Box 10.2: Why citation counts are often misleading as a marker of quality.

Addressing some of these criticisms, the Journal Citation Reports introduced a number of augmentations in 2007, such as the five-year journal impact factor and Eigenfactor. The five-year impact factor score is similar in nature to the traditional two-year impact factor but deals with a five-year citation window, which can be more useful for research areas in which articles are published and cited at a slower pace. Eigenfactor is based on the structure of the scholarly citation network (based on incoming citations, weighting citations from highly ranked journals more heavily) and gives a numerical indicator of the overall contribution of a journal to the literature. Eigenfactor is influenced by the size of the journal (the more articles, the higher the score). Other journal-level metrics include an Article Influence Score and the SCImago Journal Rank.

The San Francisco Declaration on Research Assessment (DORA), published in May 2013, arose from concerns within the scientific community regarding how research output is evaluated, and how scientific literature is cited. It is signed by a broad coalition of researchers, editors, publishers, research societies, universities and funding agencies. The declaration includes a set of individual recommendations for parties involved in research assessment, as well as one general recommendation:

Do not use journal-based metrics, such as Journal Impact Factors, as a surrogate measure of the quality of individual research articles, to assess an individual scientist's contributions, or in hiring, promotion, or funding decisions. (DORA, 2013) 
DORA recommends that publishers use a variety of journal-based metrics to provide a more nuanced picture of how journals are performing. Another recommendation is to encourage a shift toward assessment based on the scientific content of an article, rather than the publication metrics of the journal (DORA, 2013). One way of promoting this shift is to provide article-level metrics, such as downloads, citation counts, and altmetrics. Altmetrics measure science dissemination more broadly than traditional research impact, looking at how articles are discussed in the news and social media, saved and bookmarked in reference management tools, and recommended in postpublication peer-review systems (such as F1000 rating) (Cheung, 2013; Leydesdorff, 2009). However, the usefulness of altmetrics is limited from a bibliometric perspective because they are difficult to standardize and some of the measures can be gamed (Priem, 2013).

Because the journal impact factor is badly suited for assessing the individual quality and quantity of scientific output by a researcher, a number of authorbased bibliometric indicators have been developed. These include indices such as the h-index, hI-index, hm-index, i10-index, n-index, several m-indices, A-index, R-index, and the g-index. The multitude of indices reflects the difficulty in developing quantitative measures for assessing the quality of research (Fersht, 2009; Jasco, 2008; West et al., 2010a, 2010b).

\section{Conclusions}

Citations are the primary formal means by which scientific findings are communicated. In terms of full transmission of information, ideally citation practice would involve comprehensive and objective use of the whole corpus of relevant published literature. Clearly this is impracticable. However, it should still be possible to approximate this ideal by adopting a few guidelines. These recognize that citation serves the dual function of enabling verification of statements and acknowledging contributions.

In the case of formal reviews, the principles are well documented: The sources searched and the search rules should be clearly specified, as should the inclusion and exclusion criteria for articles. The sources should go beyond Web of Science databases and include searching reference lists of articles in the search domain. Regarding informal reviews, such as are used to introduce research reports, the following principles can be applied:

1. Support all nonobvious, substantive claims by citation or direct evidence.

2. Do not support statements of the obvious by citation.

3. If there is an authoritative review on a well-supported statement, this may be used in place of original articles.

4. When citing original articles, cite the discovery article together with a small number of other articles that illustrate the generality of the phenomenon. 
5. Resist the propensity to do the following:

a. prefer citations from your own country of origin unless the finding in question is country specific;

b. prefer citations from yourself and colleagues;

c. limit citations to those that support a contention, when in fact there are others that conflict with it;

d. cite output that is readily retrievable if there are more appropriate references; and

e. provide an unnecessarily large number of citations for a single statement.

6. Avoid citing inaccessible sources wherever possible.

7. When using citations in support of substantive statements, either use references that have been through some kind of peer-review process or provide an appropriate caveat.

Citation counts are widely used as an index of quality. Given that few if any researchers are able to follow all the above principles, together with the many other factors that influence the number of times a piece is cited, citation counts are a highly problematic index of quality. Journal impact factors are even more problematic. Authors should be aware of this and not be beguiled by their apparent objectivity. Ultimately, there appears at present to be no substitute for peer evaluation of research output, however flawed and subjective this might be.

Please visit the website of the International Society of Addiction Journal Editors (ISAJE) at www.isaje.net to access supplementary materials related to this chapter. Materials include additional reading, exercises, examples, PowerPoint presentations, videos, and e-learning lessons.

\section{Note}

${ }^{1}$ We are grateful to Klaus Mäkelä for this insight.

\section{References}

Aksnes, D. (2003). A macro study of self-citation. Scientometrics, 56, 235-246. Bannigan, K., Droogan, J., \& Entwistle, V. (1997). Systematic reviews: What do they involve? Nursing Times, 93, 52-53.

Bloch, S., \& Walter, G. (2001). The Impact Factor: Time for change. Australian and New Zealand Journal of Psychiatry, 35, 563-568. 
Braun, T. (2003). The reliability of total citation rankings. Journal of Chemical Information and Modeling, 43, 45-46.

Callaham, M., R. L. Wears, \& Weber, E. (2002). Journal prestige, publication bias, and other characteristics associated with citation of published studies in peer-reviewed journals. JAMA 287, 2847-2850.

Campbell, F. M. (1990). National bias: A comparison of citation practices by health professionals. Bulletin of the Medical Library Association, 78, 376-382.

Chalmers, I., Enkin, M., \& Keirse, M. J. (1993). Preparing and updating systematic reviews of randomized controlled trials of health care. Milbank Quarterly, 71, 411-437.

Cheung, M. K. (2013). Altmetrics: Too soon for use in assessment. Nature, 494, 176. DOI: https://doi.org/10.1038/494176d

Cook, D. J., Sackett, D. L., \& Spitzer, W. O. (1995). Methodologic guidelines for systematic reviews of randomized control trials in health care from the Potsdam Consultation on Meta-Analysis. Journal of Clinical Epidemiology, 48, 167-171.

Covey, L. S. (1999). Tobacco cessation among patients with depression. Primary Care, 26, 691-706.

DORA. (2013). San Francisco Declaration on Research Assessment: Putting science into the assessment of research. Retrieved from http://www.ascb.org/ dora-old/files/SFDeclarationFINAL.pdf.

Fassoulaki, A., Papilas, K., Paraskeva, A., \& Patris, K. (2002). Impact factor bias and proposed adjustments for its determination. Acta Anaesthesiologica Scandinavica, 46, 902-5.

Fassoulaki, A., Paraskeva, A., Papilas, K., \& Karabinis, G. (2000). Self-citations in six anaesthesia journals and their significance in determining the impact factor. British Journal of Anaesthesia, 84, 266-269.

Fersht, A. (2009). The most influential journals: Impact Factor and Eigenfactor. PNAS, 69, 6883-6884.

Fowler, J. H., \& Aksnes, D. W. (2007). Does self-citation pay? Scientometrics, 72, 427-437.

Gallagher, E. J., \& Barnaby, D. P. (1998). Evidence of methodologic bias in the derivation of the Science Citation Index impact factor [see comments]. Annals of Emergency Medicine, 31, 83-86.

Glassman, A. H., Helzer, J. E., Covey, L. S., Cottler, L. B., Stetner, F., Tipp, J. E., \& Johnson, J. (1990). Smoking, smoking cessation, and major depression. JAMA, 264, 1546-1549.

Grange, R. I. (1999). National bias in citations in urology journals: Parochialism or availability? [see comments]. BJU International, 84, 601-603.

Hecht, F., Hecht, B. K., \& Sandberg, A. A. (1998). The journal "impact factor": A misnamed, misleading, misused measure. Cancer Genetics and Cytogenetics, 104, 77-81. 
Hitsman, B., Borrelli, B., McChargue, D. E., Spring, B., \& Niaura, R. (2003). History of depression and smoking cessation outcome: A meta-analysis. Journal of Consulting and Clinical Psychology, 71, 657-663.

Jasco, P. (2008). The Pros and Cons of Computing the H-index Using Web of Science. Online Information Review, 32, 673-688.

Jasco, P. (2009). Five-year impact factor data in the Journal Citation Reports. Online Information Review 33, 603-614.

Jemec, G. B. (2001). Impact factors of dermatological journals for 1991-2000. BMC Dermatology, 1, 7.

Jones, A. W. (1999). The impact of Alcohol and Alcoholism among substance abuse journals. Alcohol and Alcoholism, 34, 25-34.

Kjaergard, L. L., \& Gluud, C. (2002). Citation bias of hepato-biliary randomized clinical trials. Journal of Clinical Epidemiology, 55, 407-410.

Lancho Barrantes, B. S., Bote, G., Vicente, P., Rodríguez, Z. C., \& de Moya Anegón, F. (2012). Citation flows in the zones of influence of scientific collaborations. Journal of the American Society for Information Science and Technology, 63, 481-489.

Leydesdorff, L. (2009). How are new citation-based journal indicators adding to the bibliometric toolbox? Journal of the American Society for Information Science and Technology, 60, 1327-1336.

Moher, D., Liberati, A., Tetzlaff, J, Altman, D. G., \& The PRISMA Group. (2009). Preferred Reporting Items for Systematic Reviews and Meta-Analyses: The PRISMA Statement. PLoS Med, 6, e1000097.

Ojasoo, T., Maisonneuve, H., \& Matillon, Y. (2002). [The impact factor of medical journals, a bibliometric indicator to be handled with care] [article in French]. Presse Médicale, 31, 775-781.

Opthof, T. (1997). Sense and nonsense about the impact factor. Cardiovascular Research, 33, 1-7.

Pasterkamp, G., Rotmans, J. I., de Klein, D. V. P., and Borst, C. (2007) Citation frequency: A biased measure of research impact significantly influenced by the geographical origin of research articles. Scientometrics, 70, 153-165.

Petticrew, M., \& Roberts, H. (2008). Systematic reviews in the social sciences: A practical guide. John Wiley \& Sons.

Priem, J. (2013). Beyond the paper. Nature, 495, 437-440.

Reeves, S., Koppel, I., Barr, H., Freeth, D., \& Hammick, M. (2002). Twelve tips for undertaking a systematic review. Medical Teacher, 24, 358-363.

Reyes, H. (2001). [The references in articles published in biomedical journals] [article in Spanish]. Revista Médica de Chile, 129, 343-345.

Rostami-Hodjegan, A., \& Tucker, G. T. (2001). Journal impact factors: A 'bioequivalence' issue? British Journal of Clinical Pharmacology, 51, 111-117.

Seglen, P. O. (1997). Why the impact factor of journals should not be used for evaluating research. BMJ 314, 498-502.

Semenzato, G., \& Agostini, C. (2000). The impact factor: Deeds and misdeeds [Editorial]. Sarcoidosis, Vasculitis, and Diffuse Lung Diseases, 17, 22-26. 
Stroup, D. F., Berlin, J. A., Morton, S. C., Olkin, I., Williamson, G. D., Rennie, D., . . . \& Thacker, S. B. (2000). Meta-analysis of observational studies in epidemiology: a proposal for reporting. Jama, 283, 2008-2012.

Sutton, A. J., Jones, D. R., Abrams, K. R., Sheldon, T. A., \& Song, F. (1999). Systematic reviews and meta-analysis: A structured review of the methodological literature. Journal of Health Services Research \& Policy, 4, 49-55.

Walter, G., Bloch, S., Hunt, G., \& Fisher, K. (2003). Counting on citations: A flawed way to measure quality. Medical Journal of Australia, 178, 280-281.

West, J. D., Bergstrom, T. C., \& Bergstrom, C. T. (2010a). Big Macs and Eigenfactor scores: Don't let correlation coefficients fool you. Journal of the American Society for Information Science \& Technology, 61, 1800-1807.

West, J. D., Bergstrom, T. C., \& Bergstrom, C. T. (2010b). The Eigenfactor metrics: A network approach to assessing scholarly journals. College \& Research Libraries, 71, 236-244.

West, R., \& McIlwaine, A. (2002). What do citation counts count for in the field of addiction? An empirical evaluation of citation counts and their link with peer ratings of quality. Addiction, 97, 501-504.

Wilhite, A. W., \& Fong, E. A. (2012). Coercive citation in academic publishing. Science, 335, 542-543. 
